\title{
High-Throughput Analysis of Urban Textures using Methods from Molecular Simulation
}

\author{
Ryan Rusali \\ Gerald J. Wang \\ Department of Civil and Environmental Engineering, Carnegie Mellon University \\ Pittsburgh, Pennsylvania
}

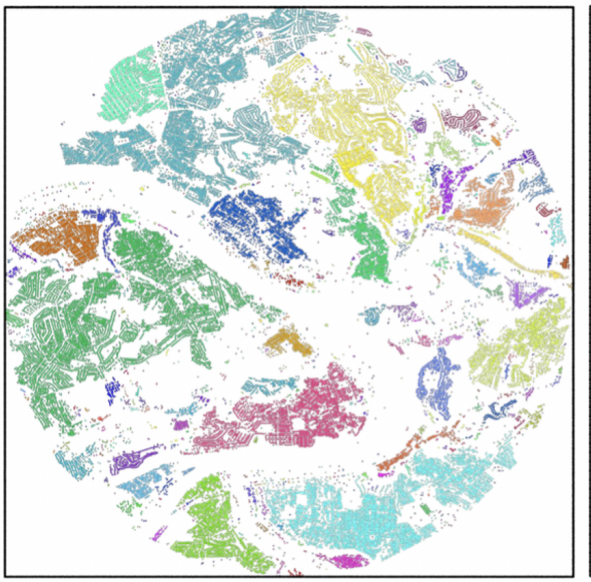

Pittsburgh

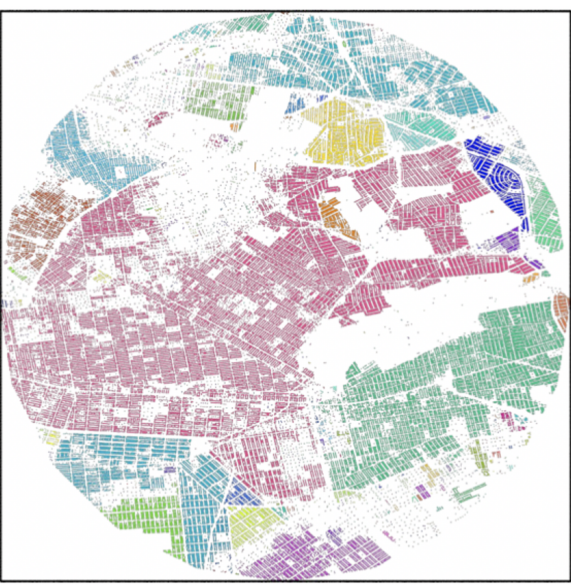

New York City

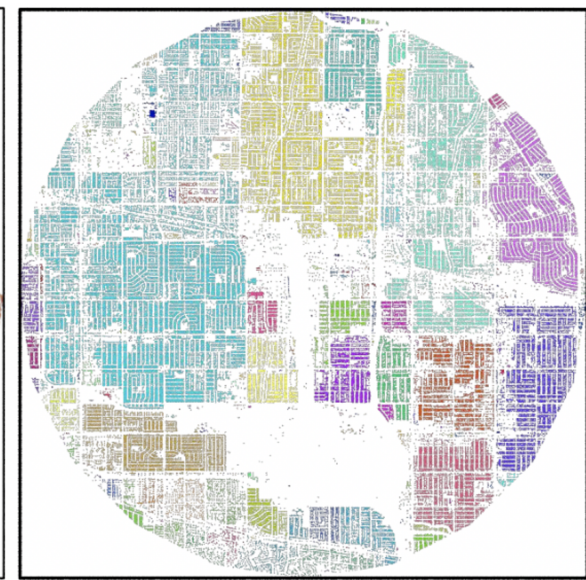

Los Angeles

Figure 1: Cluster analysis of buildings in three American cities, revealing different levels of city "crystallinity."

\begin{abstract}
A central pursuit of micro- and nano-scale engineering is describing the small-scale structure of materials in terms of spatial relationships between positions of particles (representing, e.g., individual atoms). In order to carry out such analyses, molecular simulation practitioners have developed a large array of fast methods to compute spatial relationships on particle-resolved data sets. We have developed a computational workflow that directly applies molecular simulation methods to GIS building data, in which individual buildings (instead of, e.g., atoms) are treated as the particles of interest. In so doing, we enable efficient quantification of "urban textures" consisting of $\gg O\left(10^{3}\right)$ buildings. This interdisciplinary toolkit potentially opens the door to new vistas for urban-systems modeling. As one of a few early examples, we provide evidence for a novel scaling relationship between size and ordinal rank of building clusters within several American cities, reminiscent of Zipf's Law. This scaling relation suggests a new perspective on fractal-like organization of urban environments.
\end{abstract}

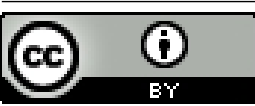

This work is licensed under a Creative Commons Attribution International 4.0 License. BuildSys '20, November 18-20, 2020, Virtual Event, Japan

(C) 2020 Copyright held by the owner/author(s).

ACM ISBN 978-1-4503-8061-4/20/11_..\$15.00

https://doi.org/10.1145/3408308.3427984

\section{CCS CONCEPTS}

- Applied computing $\rightarrow$ Physical sciences and engineering.

\section{KEYWORDS}

city structure, urban texture, molecular simulation

\section{ACM Reference Format:}

Ryan Rusali and Gerald J. Wang. 2020. High-Throughput Analysis of Urban Textures using Methods from Molecular Simulation. In The 7th ACM International Conference on Systems for Energy-Efficient Buildings, Cities, and Transportation (BuildSys '20), November 18-20, 2020, Virtual Event, Japan. ACM, New York, NY, USA, 4 pages. https://doi.org/10.1145/3408308.3427984

\section{INTRODUCTION}

Cities possess exquisite structure over a wide range of lengthscales [4]. In order to quantify - and ultimately draw useful engineering insights from - such intricate, multi-scale "urban textures," it is critical to develop tools that can process and identify patterns at all of the lengthscales of our built environment, ranging from individual buildings, to neighborhoods, to postal codes, to metropolitan areas and beyond. Significant amounts of data are required to describe a city down to the lengthscale of buildings or even blocks: New York City, for example, has $O\left(10^{5}\right)$ blocks and $O\left(10^{6}\right)$ buildings [9]. Such scale necessitates computationally efficient analysis methods.

In this work, we present an interdisciplinary approach to filling these needs, by adapting tools from the toolkit of atomic- and molecular-scale engineers. We describe a computational workflow 
that enables automated and high-throughput analysis of urban textures; here, by "texture," we specifically refer to spatial patterns and correlations in building footprints within a birds-eye view of a city. Our workflow makes use of state-of-the-art packages for processing particle data sets. This project represents a meaningful advance for a convergent approach to urban-systems modeling; it joins the domain questions and insights of city-scale engineers with the computational expertise of the nanoscale simulation community.

More broadly, this work is part of a nascent effort to study urban systems through the lens of statistical physics. This approach has yielded non-traditional and exciting insights into a wide variety of urban phenomena, including urban sprawl, population dynamics within cities, and growth of cities over time (see, e.g., the reviews by Barthelemy [2,3]). Of particular relevance to the work at hand are recent efforts to quantify the "microstructure" of a city at the level of individual buildings. Sobstyl et al. recently presented evidence of correlations between the magnitude of a city's urban heat island effect and quantitative measures of that city's "microstructural" texture [16], suggesting that detailed knowledge of texture can be used to predict (and potentially leveraged to reduce the severity of) urban heat islands. Viewed collectively, these findings strongly motivate the value of computational tools that can efficiently cast traditional sources of data on urban environments into representations amenable to the tools of statistical physics.

\section{BRIEF BACKGROUND ON STATISTICAL PHYSICS}

Although we could not possibly provide even a shadow of a meaningful synopsis of statistical physics in this section, we provide the bare essentials needed to understand the results and discussion that follow. To the interested reader, we recommend the following texts, a subset of many detailed treatments of statistical physics [8] and computer simulation of condensed matter $[1,5]$.

Throughout this section, we describe quantities that are uniquely defined given spatial coordinates for a collection of particles. In the nanoscale context, each particle typically represents the centerof-mass of an individual atom; in the city-scale context, we will interpret each particle as representing an individual building.

\subsection{Radial Distribution Function (RDF)}

In any system of particles, the RDF $g(r)$ provides a normalized sense of the number of particles roughly located at a distance $r$ from a reference particle (by "normalized," we mean that $g(r)$ is adjusted for the increased number of particles expected at larger $r$ purely due to dimensional scaling). We define $g(r)$ (in two spatial dimensions) as follows: $g(r)=\lim _{\Delta r \rightarrow 0} \frac{N(r+\Delta r)-N(r)}{2 \pi r \cdot \Delta r \cdot \sigma}$, where $N(r)$ is the number of particles we would find, on average, within a circle of radius $r$ around a reference particle, and $\sigma$ is the surface density of particles. It is worth noting that the denominator of $g(r)$ is defined such that in the limit $r \rightarrow \infty, g(r)$ approaches unity. In the study of condensed matter, $g(r)$ is venerated as a sort of holy grail, as almost all structural descriptors can be obtained from $g(r)$.

\subsection{Connectivity-Based Clustering}

Given a set of particles and a neighbor distance $d$, we can construct a graph $G$ (unweighted and undirected) by treating each particle as a node and drawing an edge between two particles iff their separation distance is less than $d$. Given this graph, we define the connectivitybased clusters of the system to be the connected sub-graphs within $G$. In Fig. 1, each cluster is assigned a unique color.

\subsection{Orientational Order Parameter}

Given a set of particles and the corresponding graph edges described above, based on the neighbor distance $d$, we define the $c$-fold orientational order parameter $\psi_{c}$ as follows: $\psi_{c}(j)=\left\|\frac{1}{n} \sum_{k=1}^{n} \exp \left(i c \theta_{j k}\right)\right\|^{2}$, where $n$ is the number of particles sharing an edge with particle $j$, $i \equiv \sqrt{-1}$, and $\theta_{j k}$ is the angle between the vector $\vec{r}_{j k}$ (connecting particles $j$ and $k$ ) and the vector $(1,0)$. This quantity is bounded from below by 0 ("complete disorder") and from above by 1 ("perfect $c$-fold rotational symmetry").

\section{COMPUTATIONAL METHODS}

We now provide an outline for our computational workflow. Our data are initially drawn from municipal GIS databases containing building footprints (we use data from, e.g., [7, 11, 12]). The data is imported into QGIS [14], which is used to compute the 2D centroid of each building's footprint. We treat this set of centroids as our system of particles; for the present analyses, we do not use any other information about each building besides its centroid. Finally, we format these files as plain text amenable to the molecular simulation analysis packages OVITO [17] and Freud [15], which are used to calculate quantities such as $g(r)$ or $\psi_{c}$. We have developed scripts that automate this cycle, enabling high-throughput calculation of microstructural quantities from GIS data.

\section{RESULTS AND DISCUSSION}

\subsection{Radial Distribution of Buildings}

In Fig. 2(a), we show the values of $g(r)$ computed for two cities with remarkably different short-range order. These radial distributions share several similarities with their nanoscale counterparts. For very small values of $r(r \lesssim 1$, in units of the characteristic particle diameter), all radial distributions are uniformly zero, indicating the presence of an excluded volume around each particle. In other words, neither atoms nor buildings are allowed to overlap each other. For small values of $r(r \sim O(1)), g(r)$ exhibits distinct spikes for a crystalline phase, reflecting geometrically well defined sets of neighbors ("coordination shells"); and gentle oscillations for a fluid phase, reflecting weak local ordering effects. In this sense, whereas Los Angeles bears some resemblance to a crystalline material, Pittsburgh has a structure that is reminiscent of a fluid. This result demonstrates the viability of using RDFs as a classification tool based on quantitative differences in urban texture (e.g. grid plans vs. alternative layouts), an idea also explored in [16]. Since it is fundamentally a measure of spatial patterning in the built environment, we envision that a city's $g(r)$ could play a useful role in modeling human mobility (e.g. pedestrian or traffic flows).

\subsection{Zipf's Law... of Building Clusters?}

We now ask: What is the distribution of sizes for connectivitybased building clusters? This question allows us to quantify a sort 


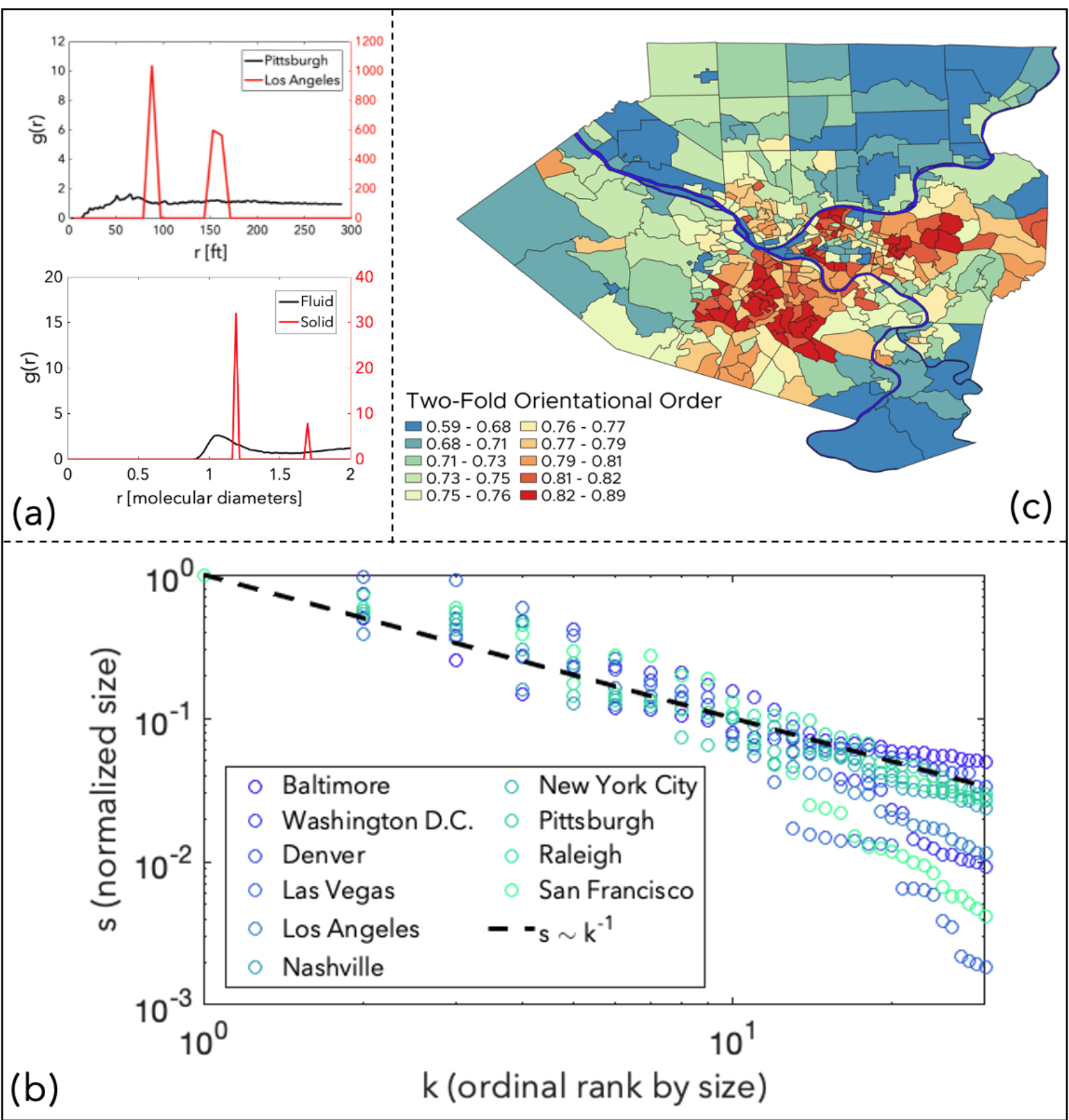

Figure 2: (a) RDFs for buildings in Pittsburgh and Los Angeles and (for the purposes of analogy and comparison) atoms in a Lennard-Jones fluid and solid; (b) Normalized size $s$ vs. ordinal rank $k$ for ten American cities, overlaid with a Zipfian power law $\left(s \sim k^{-1}\right)$; (c) Pittsburgh census tracts colored by two-fold orientational order parameter of buildings located within that census tract. 
of hierarchy of clusters that naturally emerges in, e.g., Fig. 1. These data are plotted in Fig. 2(b), where we define the "normalized size" of a cluster as the number of buildings in that cluster, divided by the number of buildings in the largest cluster of that data set; by construction, when $k=1$ (indicating the largest cluster), $s=1$. We have chosen a neighbor distance $d=125 \mathrm{ft}$, motivated by the RDFs presented in Sec. 4.1. In particular, this distance cleanly separates the first coordination shell from the second coordination shell in Fig. 2(a), for the case of the Los Angeles data. One could imagine choosing a distinct neighbor distance for each city based on cityspecific features (e.g. median residential lot size); however, for our present purposes and for the sake of parsimony, we perform this analysis with a single neighbor distance.

This analysis yields a fascinating result: These data approximately collapse onto a power-law with an exponent of -1 , a result that evokes Zipf's Law (see, e.g., [13]). This surprising observation - the first of its kind, to the best of our knowledge - suggests that the Zipfian scaling seen in sizes of cities (see, e.g., [10]) also applies to sizes of building clusters within cities. In other words, this is yet another observation of fractal-like structure in cities: One can interpret the spatial structure of a city as a collective patchwork of (much) smaller city-like entities. This result is a noteworthy intellectual curiosity, but we envision that it will have important implications for urban modeling. For example, given that this scaling is empirically supported by data from American cities that are quite different from each other (in terms of population, geography, history, evolution over time, etc.), agreement with this scaling can be used as a simple (if coarse) assessment of accuracy for models of urban development and dynamics (similar to how Zipf's Law is used in assessing models of local population growth [6]).

\subsection{Orientational Order in Cities}

Finally, we turn our attention to angular patterns formed by buildings and their neighbors. In particular, we study two-fold orientational order $\left(\psi_{2}\right)$, since most buildings in a densely developed area have two nearest neighbors. When $\psi_{2}$ is large (i.e. close to unity), each building and its two nearest neighbors are very nearly collinear, a distinguishing feature of grid-planned cities. We see in Fig. 2(c) that the average value of $\psi_{2}$ can vary widely within a city. A reader familiar with the Pittsburgh region might observe: Regions with high building (and population) density tend to have high orientational order, and these regions tend to fall along the city's three rivers; in other words, there is a correlation between a high degree of urban development and a large magnitude for the orientational order parameter. However, there is a wrinkle: In the vicinity of the three-river confluence, $\psi_{2}$ takes some of its lowest values overall, suggesting a geometric "frustration" seeded by the serpentine river paths, despite the relatively high building density in this area (downtown Pittsburgh). Based on this work, we envision the possibility for insightful correlations between orientational order and other urban networks, including water- and energy-distribution systems and transportation networks. Beyond quantifying modernday urban texture, these results also raise the alluring possibility that by studying historical GIS data sets, one might quantify the nucleation and growth of cities through orientational order.

\section{CONCLUSION}

We have developed a computational workflow that enables the efficient analysis of GIS building footprint data using tools from molecular simulation. This workflow is capable of analyzing spatial patterns for major metropolitan areas on the timescale of hours using modest computational resources (e.g. a standard laptop). By applying this workflow to several American cities, we have discovered intriguing relationships that suggest a wealth of connections between urban texture and nanoscale science. Remarkably, we have discovered a Zipfian scaling in the sizes of building clusters within a city, which parallels the scaling of sizes of cities within a country.

This work is only the tip of an iceberg. We envision these kinds of tools opening new avenues for convergent thinking that unites city-scale and atomic-scale engineering, leveraging underlying commonalities in structure, with potential implications for developing accurate models of urban growth and for engineering of transportation, water, and energy systems. For existing cities, these nanoscience-inspired methods can serve as an insightful way to categorize and diagnose urban networks; for new cities, these tools could represent an exciting avenue for rational urban design. As we enter the brave new world of machine learning and other forms of data-driven engineering, our computational workflow might also serve as a fountain of easily accessible and physically meaningful data that can be employed as model inputs. Put simply, we see an enormous amount of promise in "thinking big by thinking small."

\section{REFERENCES}

[1] Michael P. Allen and Dominic J. Tildesley. 1989. Computer Simulation of Liquids. Oxford University Press.

[2] Marc Barthelemy. 2019. Modeling cities. Comptes Rendus Physique 20, 4 (2019), 293 - 307. https://doi.org/10.1016/j.crhy.2019.05.005

[3] Marc Barthelemy. 2019. The statistical physics of cities. Nature Reviews Physics 1, 6 (2019), 406-415.

[4] Michael Batty and Paul Longley. 1994. Fractal Cities: A Geometry of Form and Function. Academic Press Professional.

[5] D. Frenkel and B. Smit. 2002. Understanding Molecular Simulation. Academic Press.

[6] Xavier Gabaix. 1999. Zipf's Law for Cities: An Explanation. The Quarterly fournal of Economics 114, 3 (1999), 739-767. http://www.jstor.org/stable/2586883

[7] Allegheny County Geographic Information Systems Group. 2020. Allegheny County Building Footprint Locations. https://data.wprdc.org/dataset/alleghenycounty-building-footprint-locations

[8] J.-P. Hansen and I. R. McDonald. 2006. Theory of Simple Liquids. Elsevier.

[9] William B. Helmreich. 2013. The New York Nobody Knows: Walking 6,000 Miles in the City. Princeton University Press.

[10] Tomoya Mori, Tony E. Smith, and Wen-Tai Hsu. 2020. Common power laws for cities and spatial fractal structures. Proceedings of the National Academy of Sciences 117, 12 (2020), 6469-6475.

[11] NYC Department of Information Technology Telecommunications. 2020. NYC Building Footprints. https://data.cityofnewyork.us/Housing-Development/ Building-Footprints/nqwf-w8eh

[12] City of Los Angeles Open Data. 2014. Los Angeles Building Outlines. https: //geohub.lacity.org/datasets/813fcefde1f64b209103107b26a8909f_0

[13] David M. W. Powers. 1998. Applications and Explanations of Zipf's Law. In New Methods in Language Processing and Computational Natural Language Learning. https://www.aclweb.org/anthology/W98-1218

[14] QGIS. 2020. QGIS Geographic Information System. Open Source Geospatial Foundation Project. http://qgis.org

[15] V. Ramasubramani, B. D. Dice, E. S. Harper, M. P. Spellings, J. A. Anderson, and S. C. Glotzer. 2020. freud: A Software Suite for High Throughput Analysis of Particle Simulation Data. Computer Physics Communications 254 (2020), 107275.

[16] J. M. Sobstyl, T. Emig, M. J. Abdolhosseini Qomi, F.-J. Ulm, and R. J.-M. Pellenq. 2018. Role of City Texture in Urban Heat Islands at Nighttime. Phys. Rev. Lett. 120 (Mar 2018), 108701. Issue 10. https://doi.org/10.1103/PhysRevLett.120.108701

[17] Alexander Stukowski. 2010. Visualization and analysis of atomistic simulation data with OVITO-the Open Visualization Tool. MODELLING AND SIMULATION IN MATERIALS SCIENCE AND ENGINEERING 18, 1 (JAN 2010). 